\title{
Comparative analysis of decametre "drift pair" bursts observed in 2002 and 2015
}

\author{
Ya.S. Volvach ${ }^{1 *}$, A. A.Stanislavsky ${ }^{1,2}$, A. A. Konovalenko ${ }^{1}$, A. A. Koval ${ }^{3}$, V. V. Dorovskyy ${ }^{1}$ \\ ${ }^{1}$ Institute of Radio Astronomy of NAS of Ukraine, Mystetstv St., 4, 61002, Kharkiv, Ukraine \\ ${ }^{2}$ V. N. Karazin Kharkiv National University, Svobody Sq., 4, 61022, Kharkiv, Ukraine \\ ${ }^{3}$ Institute of Space Sciences, Shandong University, 264209, Weihai, China
}

\begin{abstract}
We report about new observations of solar "drift pair" (DP) bursts by means of the UTR-2 radio telescope at frequencies 10-30 MHz. Our experimental data include both "forward" and "reverse" bursts with high frequency and time resolution. The records of 301 bursts, observed in 10-12 July of 2015, are investigated. The main properties of these bursts (frequency bandwidth, central frequency and others) have been analysed. In this report our main attention is paid to the comparison of our observations with the similar observations of decametre DPs performed earlier during 13-15 July of 2002 in the same frequency range. Common features of DPs in the two different pieces of data samples have been found. This may indicate the possible presence of stability in the frequency-time properties of decametre DPs from one cycle of solar activity to another.
\end{abstract}

Key words: Sun: corona, radio radiation, methods: observational

\section{INTRODUCTION}

Solar "drift pair" bursts were discovered by Roberts in 1958 [7]. DPs are detected by groundbased instruments at decametre (starting with ionospheric cut-off) and metre wavelengths (up to $80 \mathrm{MHz}$ ). Their characteristic features lie in a special morphological form on dynamic spectra of radio records, namely they consist of two narrow components separated in time, often the second one being the repetition of the first [2]. These bursts are limited in frequency bandwidth and drift in frequency [6]. Furthermore, their frequency drift rate may be both positive and negative $[2,4,7]$. Typically, DPs are observed during the solar storms of type III bursts, but not every storm leads to DPs [4]. The polarization measurements of DPs show the significant difference in degree of polarization between the two components of a pair [10]. Different theories have been proposed in literature (see their discussion, e. g., in $[5,6]$ ), but unfortunately they explain only a part of properties specific to DPs. The further development of the existing theories or making a new theory, explaining all the DP features self consistently, is an actual problem for a separate consideration.

As it was mentioned before, DPs do not occur in solar observations as often as the type III bursts. Recall that the type III bursts are the largest population of solar bursts. They can be both singles and groups. A lot of them occur during solar storms, some of which are accompanied by DPs. This explains partly why the study of DPs left more questions than answers. The last comprehensive studies of DP properties from the observations of solar radio emission in July of 2002 were presented in [5]. In the present work we compare their results with our data obtained from observations in 2015. This investigation will be a supplement and update to the previous results found in decametre wavelengths range.

\section{OBSERVATIONS}

The observations of DPs were carried out by the T-shaped Radio Telescope UTR-2 [1] during two summer campaigns in 2002 and 2015. The instrument $\left(49^{\circ} 39^{\prime} \mathrm{N}, 36^{\circ} 56^{\prime} \mathrm{E}\right)$ is located near Kharkiv, Ukraine. In 2002 three sections (instead of 12 available) of the UTR-2 were used to get a beam of $\sim 1^{\circ} \times 13^{\circ}$. The radio data were recorded independently by the analogue multichannel receiver (10$30 \mathrm{MHz}$ ), tuned into selected 60 frequencies with the frequency bandwidth $10-12 \mathrm{kHz}$ in each frequency channel, and by DSP (Digital Spectral Polarimeter), carried out the fast Fourier analysis in the continuous frequency band $17.6-29.8 \mathrm{MHz}$ with frequency of $12 \mathrm{kHz}$ and time resolution of $100 \mathrm{~ms}$ [3]. The frequency gaps between neighbour frequency channels in the 60-channel spectrometer ranged from $110 \mathrm{kHz}$ to $1.4 \mathrm{MHz}$ (most of them had $300 \mathrm{kHz}$ in frequency spacing) depending on the radio interference environment at $10-30 \mathrm{MHz}$. Due to a complex and changeable interference "weather" in decametre wavelengths, especially in the daytime, the receivers of this spectrometer were slightly tunable from time to time.

During the solar observations of 2015, the antenna configuration was similar to the previous one, four sections of the north-south array. In this case

\footnotetext{
*yarvolvach@ukr.net

(C) Ya.S. Volvach, A. A.Stanislavsky, A. A. Konovalenko, A. A. Koval, V. V. Dorovskyy, 2016
} 
the solar radio emission was received from the antenna by a new recorder, namely the digital receiver/spectrometer operating in the frequency range of $9-33 \mathrm{MHz}$ with the time resolution of $50 \mathrm{~ms}$ and the frequency resolution of $4 \mathrm{kHz}$. Recently this device for various radio astronomy applications has been developed and manufactured at the Institute of Radio Astronomy of the National Academy of Sciences of Ukraine [8]. Notice that its appearance in the UTR-2 observatory has improved the reliability of obtained data.

According to [5], during the daily observations from 6:00-12:00 UT on 13-15 July of 2002, there has been detected about 774 DPs including both "forward" (FDP) and "reverse" (RDP) bursts. In this case only 198 bursts, recorded on 13 July of 2002 using the DSP, were subjected to a detailed analysis of all the parameters, such as the duration of the first and second components, the time delay between DP components, instantaneous bandwidth and radiation flux. Only two parameters from the DP data obtained by the 60-channel spectrometer were analysed. They are central frequencies and frequency drift rates of DP components. In the solar observations on 10-12 July of 2015 we have found 301 DP bursts, 92 of which were RDPs, and 209 FDPs. All of them were recorded by the digital receiver/spectrometer at 9-33 MHz.

\section{RESULTS}

One of the most important characteristics of DPs is its distribution over the frequency range. It determines the number of events at distinct frequencies with the step of $1 \mathrm{MHz}$. Fig. 1 shows the corresponding histograms for the DPs data obtained in 2002 and 2015. As it is easily seen, the number of FDPs decreases at high frequencies, whereas the number of RDPs is reduced to lower frequencies. A difference between the DP data of 2002 and 2015 should be noted. At low frequencies of the frequency band the contribution of FDPs is underrepresented in the observations of 2002, and this is not the same in the FDPs histogram of 2015. A similar situation is observed for RDPs at high frequencies. This may be explained by the fact that the bursts of 2002, trapped partly in the frequency range of observations (not completely visible on the dynamic spectrum), were excluded from consideration.

In the observations of 2015 all the DP bursts without exception were taken into account. Therefore, the histograms of 2015 indicate that the number of RDPs increases in the direction of high frequencies, and FDPs seek on the other direction of the frequency range. This fact can be characterized by skewness as a measure of asymmetry of the distributions. FDPs have 0.237 whereas it is -0.966 for RDPs. Recall here that negative skew indicates a longer tail of the distribution on the left side comparing to the right side, and positive skew - longer distribution tail on the right. Unfortunately, the frequency range of DP observations is not wide enough to characterize the distributions of FDPs and RDPs completely. Observations at lower frequencies are needed for the studying FDPs, but there is the ionospheric cut-off for ground-based instruments, while the space-based observations do not give an acceptable resolution in time and frequency. Besides, their instrumentation sensitivity is not high enough. On the other hand, observation at higher frequencies, that are required for the analysis of RDPs, have better prospects with the development of a new lowfrequency radio telescopes in the present days (see, e. g., [9] and references therein).

Another interesting DP parameter is the total frequency bandwidth occupied by such bursts. We have analysed this parameter for FDPs and RDPs using observational data sample of 2015. Fig. 2 presents their frequency bandwidth distributions. According to our data the average value of frequency bandwidth is $2.82 \pm 1.32 \mathrm{MHz}$ for RDPs, and $3.6 \pm 2.4 \mathrm{MHz}$ for FDPs. Despite of the fact that the long DPs observed in 2002 were not taken into account, the average values of DPs frequency bandwidth from the recent observations are close to the values from [5], namely $4.07 \pm 1.82 \mathrm{MHz}$ and $3.17 \pm 1.65 \mathrm{MHz}$ for FDPs and RDPs, respectively. Recall that the DPs frequency bandwidth from observations of 2002 has been established by means of DSP. It is interesting to note that our observations on 2015 clearly showed that high-frequency edges of long DPs were located upward the frequency band of the UTR-2 radio telescope.

Histograms of the frequency drift rates of DPs for both, 2002 and 2015, data samples are shown in Fig. 3. Comparing these two data samples, one can notice that, basically, they have much in common. For example, the distributions of FDPs and RDPs from 2002 and 2015 data sets have similar shapes: compact distribution of the FDPs and a fat tail in the direction of high frequency drift rates for RDPs. The statistical properties of these bursts are listed in Table 1. Both data sets have similar values of means and rms. More detailed the frequency drift rates of DPs will be considered elsewhere.

Table 1: Means and rms of DPs in frequency drift rate on 2002 and 2015.

\begin{tabular}{l|rr|rr}
\hline years & \multicolumn{3}{|c}{2015} & 2002 \\
\hline type & FDPs & RDPs & FDPs & RDPs \\
\hline mean, MHz/s & -0.83 & 1.77 & -0.78 & 1.8 \\
$\mathrm{rms}, \mathrm{MHz} / \mathrm{s}$ & 0.6 & 1.1 & 0.42 & 1.6 \\
\hline
\end{tabular}

\section{CONCLUSIONS}

With good reason the study of DPs can be attributed to astrophysical problems accessible for investigations only at low radio frequencies $(\sim 10$ $100 \mathrm{MHz}$ ). The continuous modernization of the 

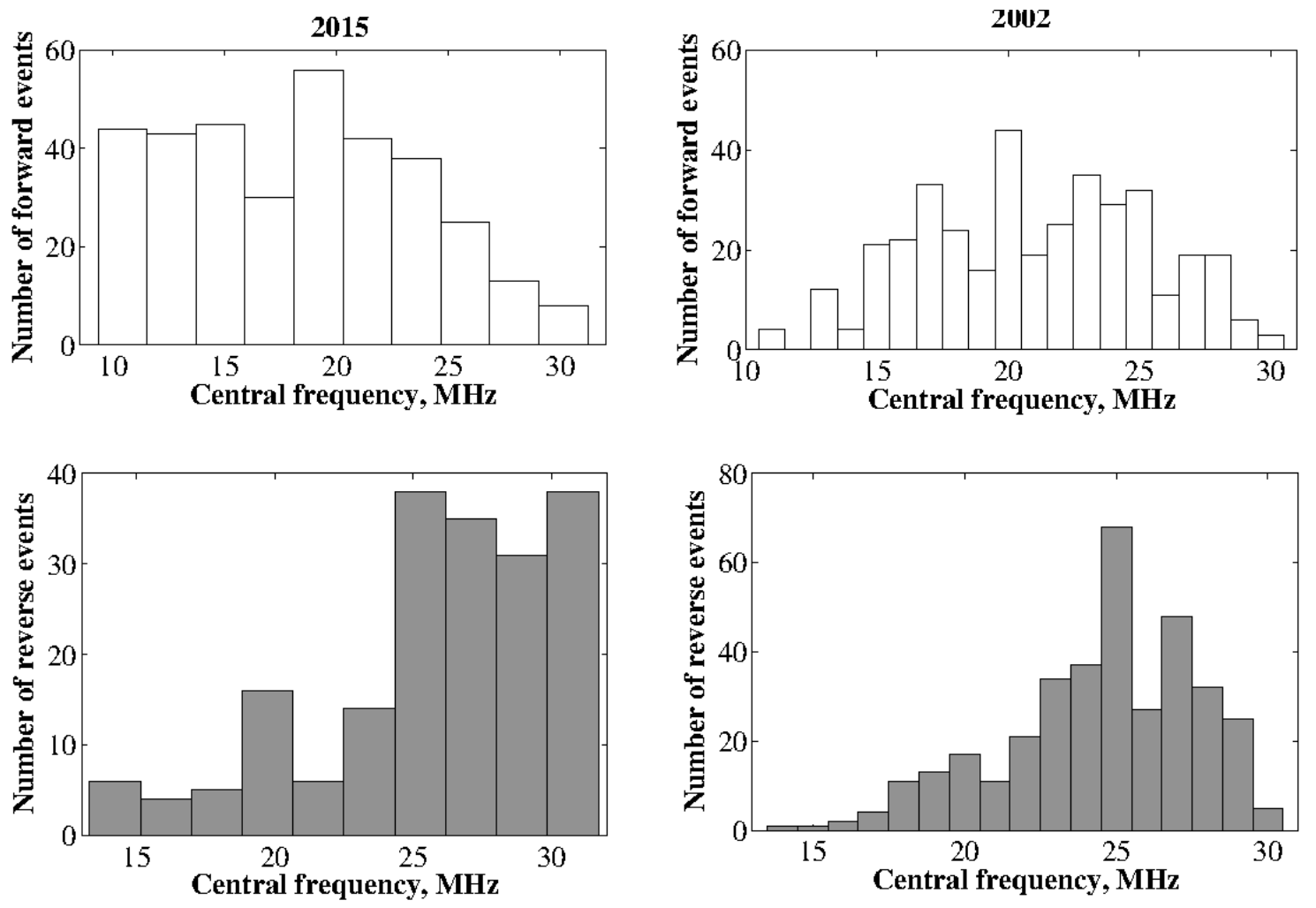

Fig. 1: Comparison of central frequency histograms obtained for FDPs and RDPs from the observations in 2002 and 2015.

UTR-2 radio telescope improves the quality of obtained results in the study of solar bursts. The present work demonstrates one of achievements in this direction. In particular, it has been shown that decametre FDPs and RDPs differ in properties. On the other hand, many similar features between these two sets of DP data (2002 and 2015) indicate the stability of the frequency-time properties of decametre DPs from one cycle of solar activity to another. In this context the capabilities of a new Ukrainian ultrabroadband radio telescope GURT (Giant Ukrainian Radio Telescope), being built now, will be extremely useful for understanding the state of DPs in the manifestation of solar activity.

\section{ACKNOWLEDGEMENT}

This research was partially supported by Research Grant "Synchronized simultaneous study of radio emission of solar system objects by lowfrequency ground- and space-based astronomy" from the National Academy of Sciences of Ukraine.

\section{REFERENCES}

[1] BraudeS. Ia., Megn A. V., Riabov B. P., Sharykin N. K. \& Zhuk I. N. 1978, Ap\&SS, 54, 3

[2] Ellis G.R. A. 1969, Australian J. Phys., 22, 177

[3] Kleewein P., Rosolen C. \& Lecacheux A. 1997, in Planetary radio emission IV, Proc. 4th International Workshop held at Graz, Austria, eds.: Rucker H.O., Bauer S. J. Lecacheux A., Austrian Academy of Sciences Press, Vienna, 349

[4] de la Noë J. \& Møller-Pedersen B. 1971, A\&A, 12, 371

[5] Melnik V. N., Konovalenko A. A., Dorovskyy V. V. et al. 2005, Solar Phys., 231, 143

[6] Møller-Pedersen B., Smith R. A. \& Mangeney A. 1978, A\&A, 70, 801

[7] Roberts J. A. 1958, Australian J. Phys., 11, 215

[8] Ryabov V. B., Vavriv D. M., Zarka P. et al. 2010, A\&A, 510, A 16

[9] Stanislavsky A. A., BubnovI. N., Konovalenko A. A. et al. 2014, Adv. Astron., 2014, ID 517058

[10] Suzuki S. \& Gary D. E. 1979, in Proc. Astron. Soc. Australia, 3, 379 

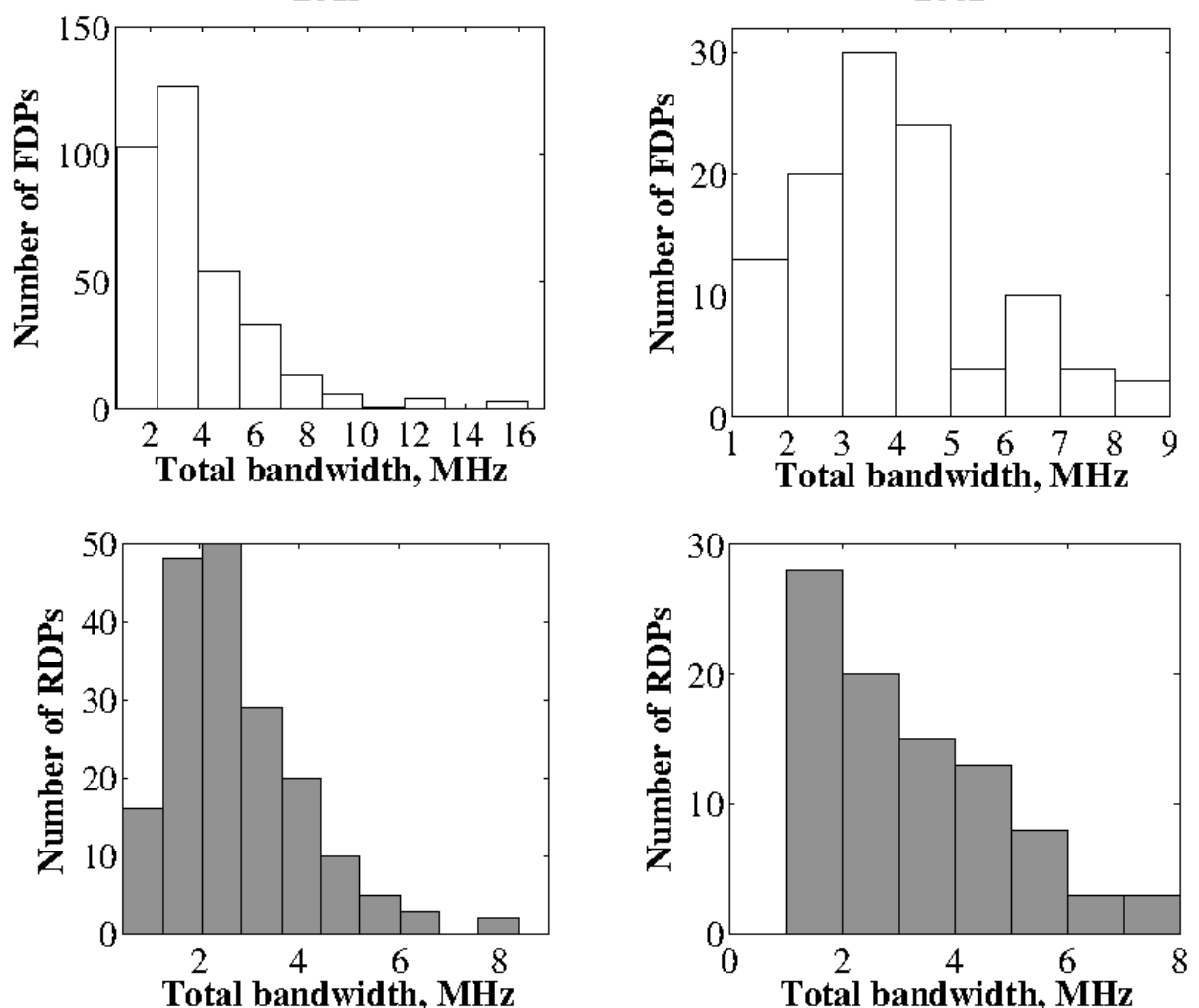

Fig. 2: Comparison of frequency bandwidth histograms obtained for FDPs and RDPs according to the observations in 2002 and 2015.

2015
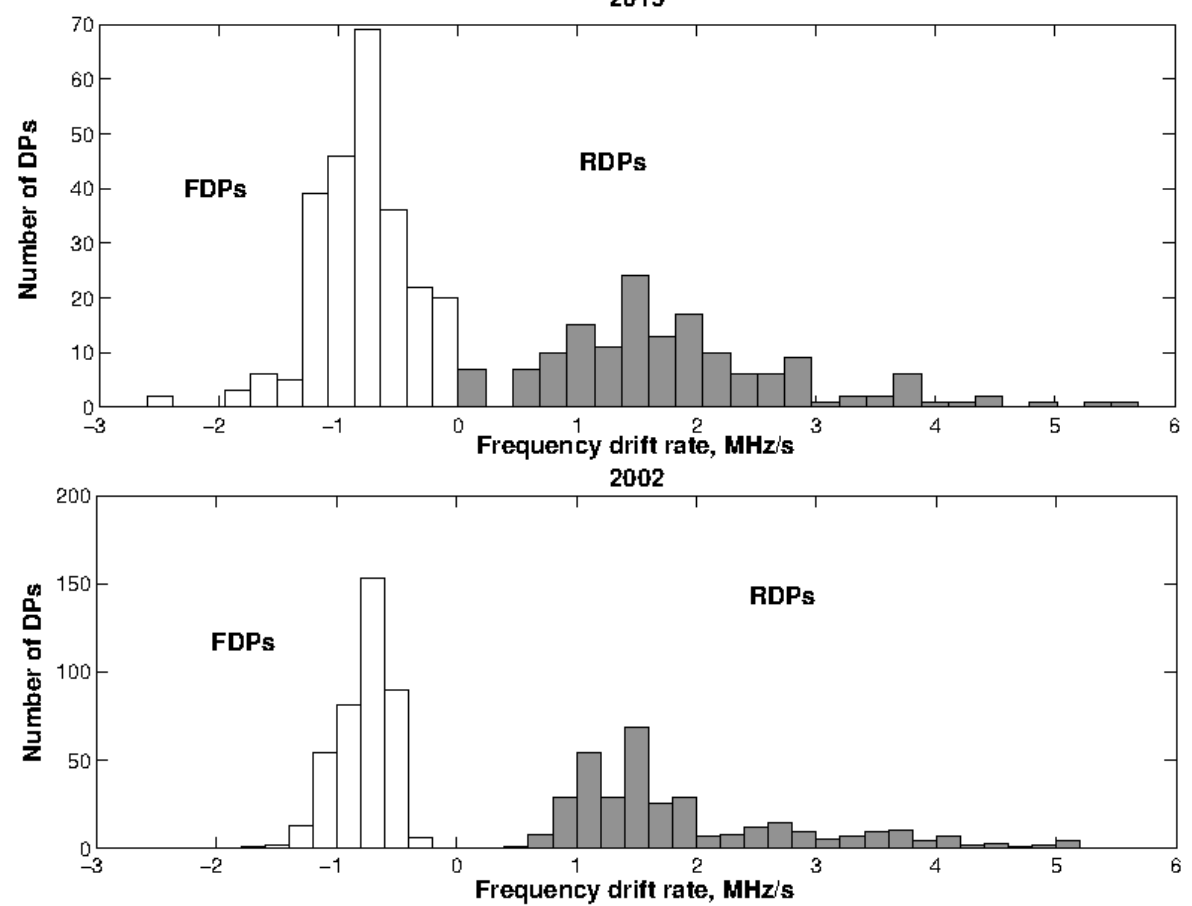

Fig. 3: Histograms of frequency drift rates on the central frequency for FDPs (white columns) and RDPs (shaded columns) in 2002 and 2015. 